\title{
Cancer survivorship research: the challenge of recruiting adult long term cancer survivors from a cooperative clinical trials group
}

\author{
Patricia A. Ganz • Stephanie R. Land - Cynthia Antonio • Ping Zheng • Greg Yothers • \\ Laura Petersen • D. Lawrence Wickerham • N. Wolmark • Clifford Y. Ko
}

Received: 15 May 2009/Accepted: 3 June 2009/Published online: 13 June 2009

(C) The Author(s) 2009. This article is published with open access at Springerlink.com

\begin{abstract}
Introduction With the growing number of adult cancer survivors, there is increasing need for information that links potential late and long term effects with specific treatment regimens. Few adult cancer patients are treated on clinical trials; however, patients previously enrolled in these trials are an important source of information about treatmentrelated late effects.

Methods Focusing on colorectal cancer survivors, we used the database from five phase III randomized clinical trials from the National Surgical Adjuvant Breast \& Bowel Project (NSABP) to recruit and enroll long term survivors in a study of late health outcomes and quality of life. We describe the challenges to recruitment of patients more than $5-20$ years after treatment.

Results Sixty-five NSABP treatment sites were invited to enroll patients in the study. Sixty participated with the potential to
\end{abstract}

\section{P. A. Ganz $(\bowtie)$}

School of Public Health \& David Geffen School of Medicine,

University of California, Los Angeles,

Los Angeles, CA, USA

e-mail: pganz@mednet.ucla.edu

P. A. Ganz $\cdot$ C. Antonio $\cdot$ L. Petersen $\cdot$ C. Y. Ko

Division of Cancer Prevention \& Control Research,

Jonsson Comprehensive Cancer Center, University of California,

Los Angeles,

Los Angeles, CA, USA

S. R. Land $\cdot$ P. Zheng $\cdot$ G. Yothers

National Surgical Adjuvant Breast \& Bowel Project (NSABP),

Biostatistical Center,

Pittsburgh, PA, USA

S. R. Land $\cdot$ G. Yothers

Department of Biostatistics, Graduate School of Public Health,

University of Pittsburgh,

Pittsburgh, PA, USA recruit 2,408 patients. We received registration forms on only 976 patients (41\%) of whom 744 (76\%) expressed interest in participating and 708 completed interviews (95\% of those expressing interest; $29 \%$ of total potential sample). There were multiple barriers to recruitment (difficulty locating patients, lack of institutional commitment, lack of patient interest).

Conclusions Patients treated on clinical trials are an important potential source for examining the late effects of cancer treatments. Retrospective recruitment has substantial limitations. In the future, mechanisms should be established for prospective long-term follow-up to identify and understand the frequency and type of late effects associated with cancer treatments.

Implications for Cancer Survivors As cancer patients are living longer, it will be important to learn from participants in clinical trials whether or not specific treatment regimens are associated with any serious late effects.

\section{L. Wickerham • N. Wolmark}

National Surgical Adjuvant Breast \& Bowel Project (NSABP), Operations Center,

Pittsburgh, PA, USA

D. L. Wickerham $\cdot$ N. Wolmark

Department of Human Oncology, Allegheny General Hospital, Pittsburgh, PA, USA

C. Y. Ko

UCLA Department of Surgery,

David Geffen School of Medicine at UCLA,

Los Angeles, CA, USA

C. Y. Ko

Department of Surgery,

West Los Angeles Veterans Affairs Medical Center,

Los Angeles, CA, USA 
Keywords Colorectal cancer survivors · Clinical trials · Recruitment barriers

There has been increasing attention called to the need for more systematic information on the late effects of cancer treatment, due to the growing number of cancer survivors and the complexity of contemporary cancer treatments. The 2005 Institute of Medicine report, From Cancer Patient to Cancer Survivor: Lost in Transition [1], called for more research to identify who is at risk for long-term sequelae and how the consequences of treatment might be mitigated. Until recently, most studies of cancer survivors have used convenience samples or tumor registry databases for recruitment [2-5]. When survivors are recruited in this way, there may be little information on the treatment exposures that occurred many years earlier, making it difficult to connect specific treatments with late effects. Furthermore, if there are dose, treatment, or scheduling factors that are responsible for a late effect, they may not be apparent without detailed treatment information.

One potential way to obtain a more accurate picture of the causal relationship between cancer treatments and late effects is to recruit cancer survivors who participated in a cancer treatment clinical trial, where the treatments were delivered according to a pre-specified protocol, and accurate treatment records retained that may explain whether the patient's late effect outcomes are linked to the prior treatment. Clinical trials often assign patients to two or more competing treatment strategies, and as a result, the survivors may be examined for differences in outcomes that could be directly related to the earlier treatment. The only serious limitation of studying survivors who participated in a cancer clinical trial is that they represent a select minority of individuals who qualified for the trial and were willing to participate. Nevertheless, the fact that treatments were assigned at random eliminates the potential biases inherent in examining long-term outcomes in either tumor registry samples or convenience cohorts.

Although long-term follow-up of patients treated on National Cancer Institute (NCI) funded clinical trials would be ideal, with collection of data on long-term functional outcomes and comorbid conditions, few resources have been available to allow this to occur prospectively. As a result, long term survivor studies that have used this mechanism to identify and assess long-term cancer survivors have largely required supplementary funding, and have not built prospective follow-up into the main trial protocol [6-9]. Thus, participants in these studies often form another "convenience sample" since not all potentially eligible patients participate; however, data on specific treatments are more accurate and certainly can provide more insight into the potential linkage between treatment and late effects.
As part of a study designed to describe the long term and late effects of treatment for colon and rectal cancers in patients treated on 5 separate clinical trials conducted by the National Surgical Adjuvant Breast and Bowel Project (NSABP), we examined the barriers to recruitment of survivors identified from the clinical trials database. Colorectal cancer (CRC) survivors account for $10 \%$ of the estimated 11.1 million cancer survivors in the United States today, with few studies on the quality of life in individuals with this cancer $[5,10,11]$. Given the limited data on longterm survivorship outcomes in CRC, we designed a study that would use the database of the NSABP to examine this group of survivors. The selected trials spanned a 20 year period in terms of enrollment, and as such could provide data on very long-term survivors who had received adjuvant therapy (radiation and chemotherapy) during different treatment eras. However, this strength also posed a challenge for locating and consenting individuals who may have lost contact with the facility that provided their cancer care. In this report we describe the procedures developed to use the cooperative group database to identify and recruit survivors, as well describe the logistical issues surrounding recruitment and enrollment of long-term survivors in this type of research. Finally, we suggest strategies to enhance the collection of late effects data from future participants in cancer clinical trials.

\section{Methods}

\section{Study design}

With external funding from the American Cancer Society, a grant entitled "Patient Reported Outcomes in Long Term Survivors with Colon and Rectal Cancer" facilitated the funding of an NSABP Protocol Study LTS-01 to investigate important issues in long-term (5+ years) colon and rectal cancer survivors, by examining three types of patientreported outcomes (quality of life, function, and symptoms) and their predictors. We used a modification of the Andersen Behavioral Model to conceptualize and identify the data to be collected, as well as the analyses to be performed [12].

To overcome inherent difficulties associated with recruiting a long-term survivor sample, this study used five previously completed NSABP colon and rectal cancer trials to identify a well-characterized cohort of survivors. These survivors were recruited from closed NSABP adjuvant therapy trials that spanned a 20 -year period. There were two rectal cancer adjuvant trials, which were the oldest of the studies included. R-02, compared adjuvant MeCCNU, vincristine, and 5-FU (MOF) with and without radiation therapy, to adjuvant leucovorin and 5-FU, with and without radiation therapy, in patients with Dukes' $\mathrm{B}$ and $\mathrm{C}$ rectal 
cancer. The trial opened in August 1987 and closed to accrual in December 1992. R-02 was closed to follow-up on April 14, 2004. R-03, compared preoperative multimodality therapy (5-FU plus leucovorin plus radiation therapy) in patients with operable rectal cancer to postoperative radiation therapy with the same chemotherapy, with accrual starting in June 1993 and closing in June 1999. Although these rectal cancer trials had low enrollment and small numbers of survivors available, so little was known about these survivors that we chose to attempt to contact them anyway.

There were three colon cancer adjuvant trials: C-05, which compared 5-FU and leucovorin with 5-FU and leucovorin plus interferon (IFN)-alpha 2a, was opened to accrual in October 1991 and was closed to accrual in February 1994; C-06, compared oral Uracil/Ftorafur (UFT) and leucovorin to 5-FU and leucovorin, and was opened to accrual in February 1997 and closed to accrual in March 1999; C-07, compared 5-FU plus leucovorin plus oxaliplatin (FLOX) to 5-FU plus leucovorin (FL) and was activated February 2000, and closed to accrual in November 2002. C-07 was a contemporary trial, with some patients just reaching the five year eligibility time point during the course of our study, and we were much more optimistic about recruiting them as a result.

Detailed clinical, treatment, and quality of life data already collected in each trial were available to conduct longitudinal analyses (baseline quality of life data were available from two colon cancer adjuvant trials). The benefit of this strategy was the accurate information about initial treatment, as well as a representative North American patient population. Other recognized challenges that were anticipated in preparing the grant and protocol were the need to obtain institutional review board (IRB) approval to approach survivors at each of the North American centers who enrolled patients on these trials. Although each institution would only need to locate and consent patients, the inertia and administrative burden in doing so was recognized, and funds were made available to the institution for obtaining IRB approval, as well as locating and consenting patients. All of the research data collection was done through centralized telephone interview, to minimize the burden on institutions. The goal of all of these administrative activities was to facilitate the successful recruitment of these long-term survivors who may not have had recent contact with the NSABP site. Figure 1
Fig. 1 Overview of study procedures reflecting collaboration between NSABP, UCLA, and the NSABP Member Institutions (clinical sites). CATI= computerassisted telephone interview.

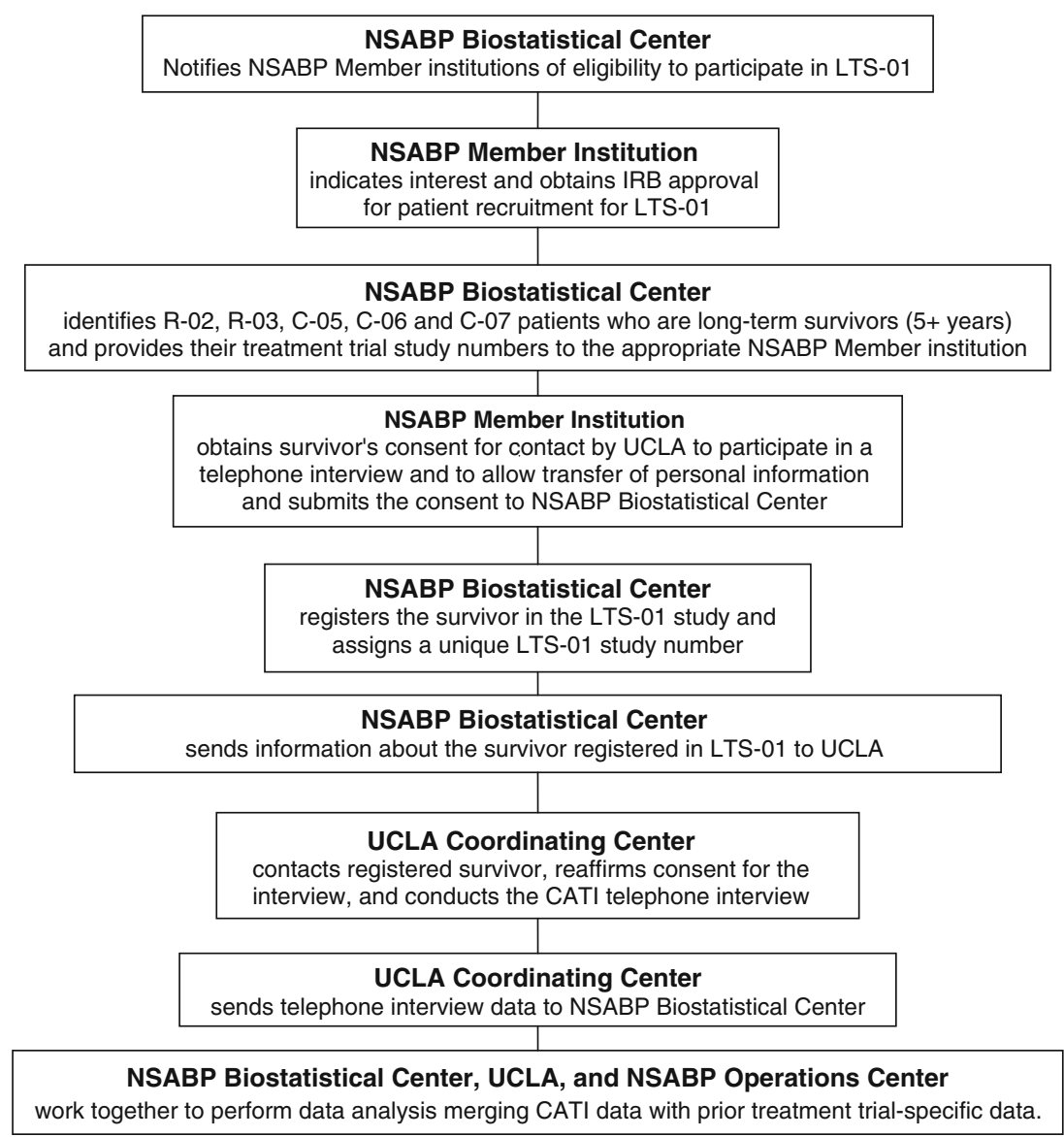


provides an overview of the procedures required to identify and consent long-term CRC survivors for this study.

Institutional recruitment

There were slightly more than 230 NSABP investigators who were providing long-term data follow-up for the CRC survivors on the five described protocols. The number of CRC survivors per investigator ranged from 1 to 110 , with a mean of 12.9 survivors/investigator. This protocol was approved for cancer control credits by the NCI Division of Cancer Prevention, and thus all CCOP sites were invited to participate, as well as the remaining top 30 accruing sites for the five trials (at least 10 potentially available survivors). To motivate institutions with a smaller number of potential participants, as well as to facilitate obtaining IRB approval, the institutions were supplied the following supporting materials:

- Model IRB application form, as well as supporting materials (e.g., copies of questionnaires, study protocol, UCLA IRB approval for the study)

- Samples of introductory letters to be mailed to potential participants

- Trifold recruitment brochure that described the study

- Telephone scripts for use by site staff to facilitate recruitment

- Model informed consent

- Model HIPAA authorization

- Access to technical support from a centralized Coordinating Center via a toll free number and e-mail correspondence.

- Centralized support to local site coordinators for assistance in IRB submission, study administration and patient recruitment

All materials were culturally sensitive to facilitate the participation of diverse populations. Due to budgetary limitations, only English speaking survivors could be included. Once IRB approval for LTS-01 recruitment and transfer of data was obtained at the local NSABP site, the investigator and staff were responsible for recruitment of eligible CRC survivors. The UCLA research team provided administrative support to the sites in helping them obtain local IRB approval for the study, as well as addressing any local challenges related to subject recruitment. Throughout the course of institutional recruitment, newsletters containing study progress information and answers to frequently asked questions were sent to institutions to encourage their participation and survivor recruitment. In addition, the LTS-01 protocol was featured both on the scientific program and in nurse-coordinator workshops at the semi-annual NSABP group meetings. Finally, targeted recruitment of some non-participating institutions, including letters to site coordinators, occurred in the final year of the study, which focused on the barriers to individual institutional participation. These issues will be discussed in detail in our results.

\section{Patient recruitment}

The anticipated sample size needed for this study across the 5 trials was 1,167 . With recruitment of these 60 institutions, we expected to have available over 2,200 survivors to approach, and we estimated a consent rate of approximately $50 \%$ based on prior studies with recruitment of long-term survivors from tumor registries. To identify survivors to approach, the NSABP Biostatistical Center generated the NSABP study numbers of potentially eligible long-term survivors from the five CRC trials linked to the respective participating NSABP investigators and sites. After receiving IRB approval, the NSABP site investigators were asked to send each identified CRC survivor an introductory letter and consent form. The letter described the study and asked for their participation. A self-addressed stamped envelope was provided for them to return a response form and the signed consent(s) to the NSABP Member institution. The response (REC) form asked the survivor the best times to be contacted for the telephone survey. If the survivor declined to participate, the reason for non-participation was collected on the form. This was also designed to learn about reasons for nonparticipation in this type of survey study. Those who did not respond to the introductory letter within three weeks were mailed a second letter. If no response was obtained after two more weeks, and if local IRB policies and procedures permitted, a follow-up phone call was made by the local NSABP site staff. If these had not been received by the subject, a new packet was mailed.

Subjects who agreed to participate in the study mailed their consent forms along with their preferred contact days and times to their local site. The local site coordinator forwarded this information to the NSABP Biostatistical Center who assigned an identification number to the subject and forwarded the information to the centralized interviewing center at UCLA. Computer assisted telephone interviews (CATI) were completed with subjects by a staff of trained interviewers. The interviewing center corresponded with the NSABP to address phone numbers that were disconnected or incorrect. In some cases, the NSABP corresponded with the local site to obtain the correct information. Up to ten attempts were made to contact subjects on their preferred day and time, as well as additional days and times if necessary. Interviewers' efforts to reach subjects included setting appointments and making calls at a convenient time including early mornings, evenings, and weekends. 


\section{Results}

Institutional recruitment

LTS-01 opened for accrual November 29, 2006, and had an anticipated recruitment period of 18 months. It was expected that it would take 3-4 months for institutions to obtain IRB approval and thereafter they would begin contacting potential participants. Figure 2 shows the cumulative rate at which IRB approval was obtained for those institutions invited to participate. Over time, as we determined the survivor recruitment was lower than expected, 5 additional selected institutions with large numbers of rectal cancer patients received invitations to participate. Figure 3 shows the results of institutional recruitment efforts as of January 15, 2009 when the trial was closed to accrual. Of the 65 institutions that were invited to participate, initially only 46 indicated interest in study activation. Of these, 45 successfully obtained IRB approval and one could not get its IRB to accept responsibility for the study and recruitment, stating that it was the responsibility of the protocol investigators conducting the interviews.

Of the 19 institutions that were initially not interested in participating, a continuous effort was made to work with these investigators and their data coordinators to overcome the barriers to obtaining IRB approval. Frequent issues that came up included: institutions perceiving a lack of available staffing or time to dedicate to the IRB process and the subsequent study; institutions perceiving a challenge to reach subjects due to having many of their eligible subjects sparsely distributed through multiple satellite sites; satellite sites lacking resources or interest to initiate their own IRB processes; institutions encountering challenges/delays in completing the IRB submission due to technical difficulties in accessing sample IRB forms or lists of eligible subjects online; general questions and concerns about the requirements of obtaining IRB approval and administering the study. Ultimately, 12 of these 19 institutions obtained IRB approval, and the closure date for the study was extended to accommodate them. Continuous monitoring of institutional participation and enrollment of the potential target patient population allowed the research team to identify high yield institutions that had not joined the study.

\section{Patient recruitment}

For the 65 institutions that were invited to participate, we identified 2,540 potentially eligible survivors to approach from the $5 \mathrm{CRC}$ adjuvant therapy trials. Figure 3 shows that among the 2,408 potentially eligible patients from the 60 participating institutions, contact was made with only 976 patients (REC forms received), representing $41 \%$ of the actual pool. Of these, 744 initially agreed to participate, for a yield of $76 \%$ of those who were contacted. However, because of our difficulty in contacting these long term survivors, the overall response rate was 708 of 2,408 or $29 \%$. Table 1 shows the distribution of demographic and
Fig. 2 Cumulative frequencies of accrued institutions with IRB approval.

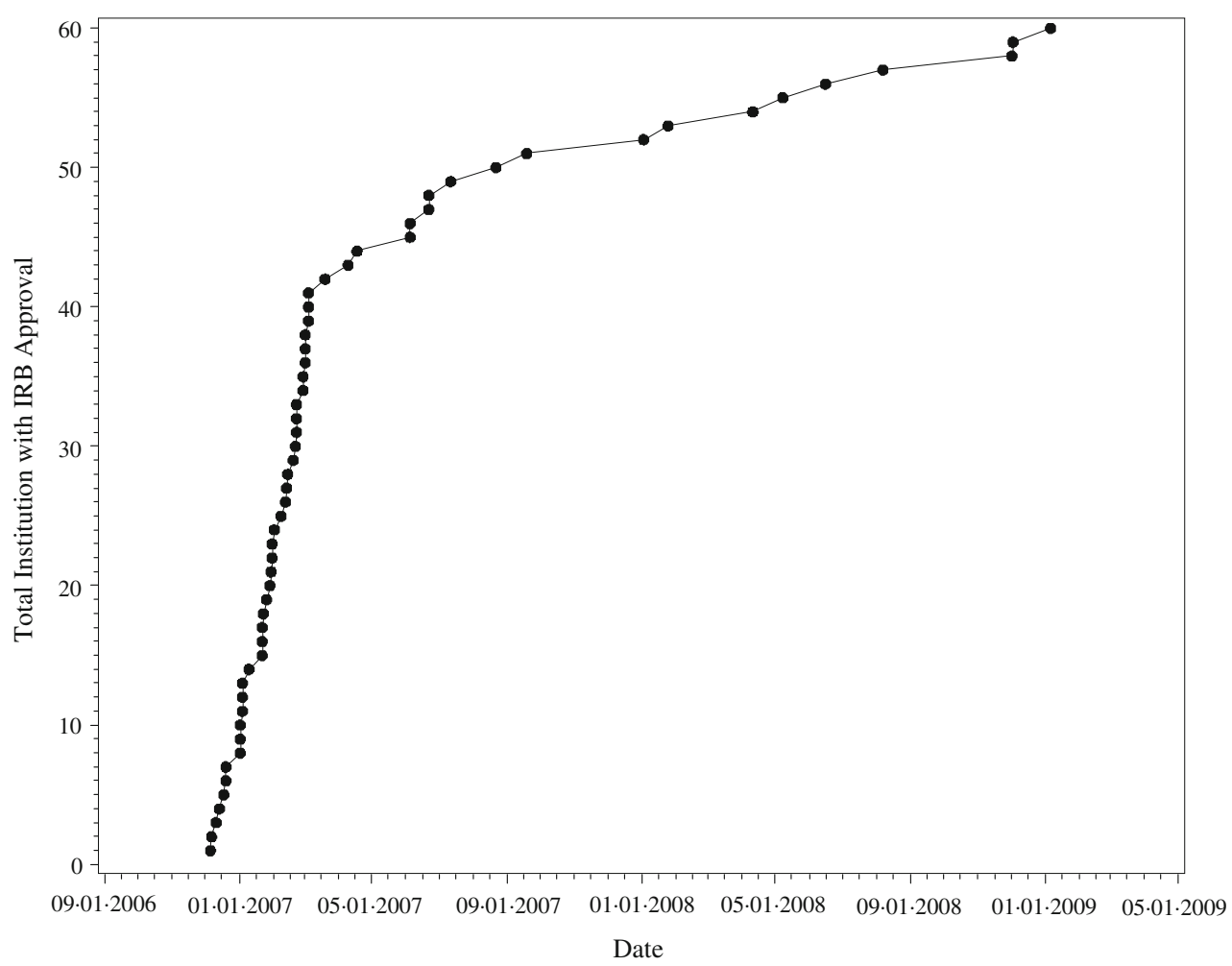




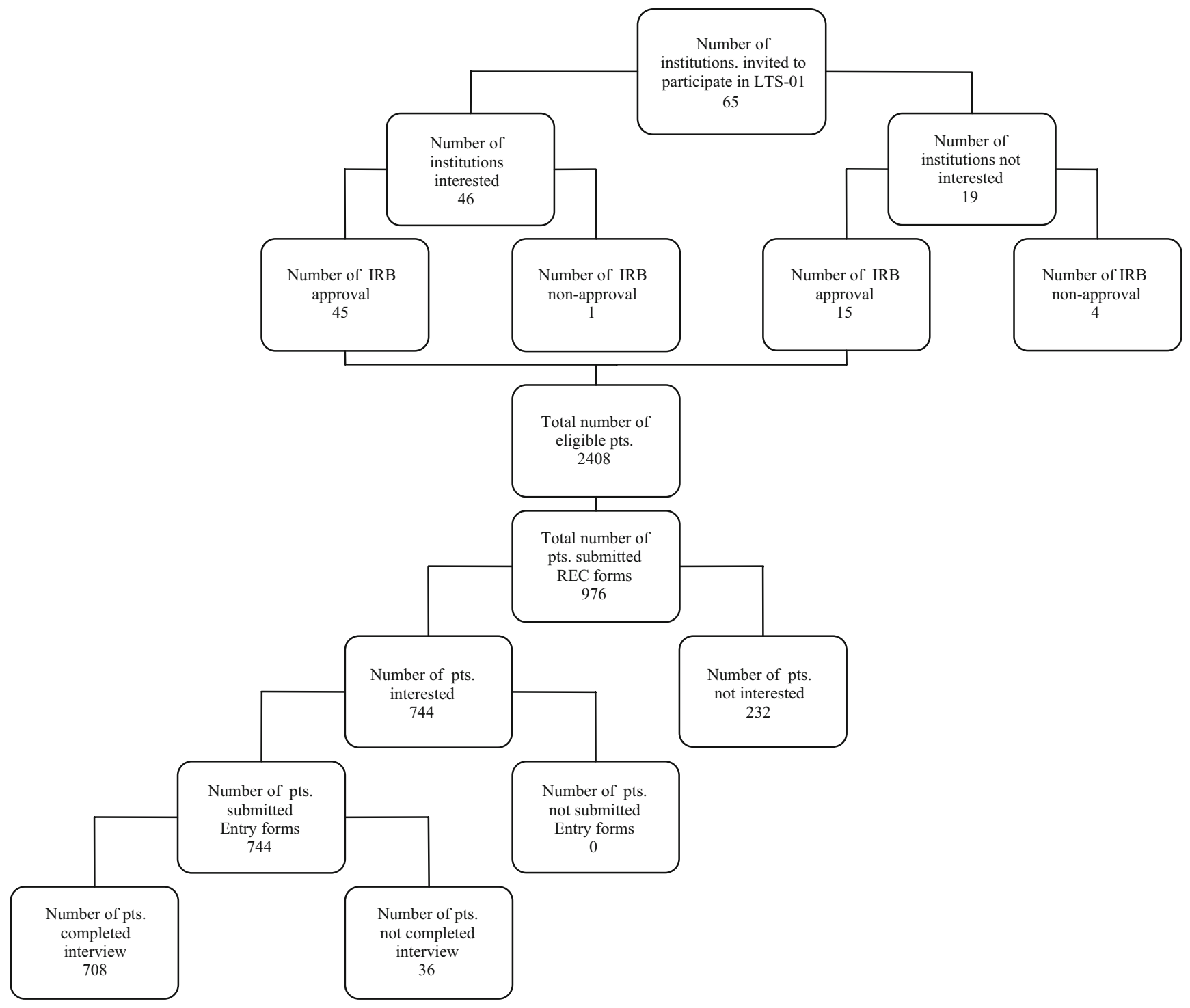

Fig. 3 Flow diagram of institutional and patient recruitment.

medical characteristics of those potentially eligible and those finally consenting to participate in this study. Men and women participated at roughly the same rate. Participants in LTS-01 were also younger and more likely to be white than the total sample who were eligible. As expected, fewer patients from the older rectal trials (R-02 and R-03) participated (only 10-16\%), and more patients from the recently completed $\mathrm{C}-07$ trial participated (33\%). These numbers were far below the target accrual of over 1,100 survivors for LTS-01.

Table 2 provides demographic data on 914 patients who were contacted and provided information about themselves, indicating whether they would participate and if not, for what reason. Those who could be contacted and were willing to participate were significantly younger [ (mean age 65.6 vs. $70.2(\mathrm{P}<0.0001)$ ], were more likely to be married $(\mathrm{P}=0.05)$, and were more likely to have been treated more recently $(\mathrm{P}=0.018)$. Reasons for not participating were recorded on this same form and are described in Table 3. The most common reason was lack of interest, followed by staff reporting that patient was either deceased (9 individuals) or unable to be located. In this elderly patient population, many were in poor health. Among those categorized as "other" included some patients with recurrent cancer, general frailty, being hard of hearing, and having difficulty locating the patient. Inability to understand and respond in English was rarely cited as a barrier.

Final recruitment through telephone interviews

In total, the UCLA coordinating center received contact information for 744 subjects and completed 708 (95\%) interviews. Upon reaching a subject, the interviewer read a script that relayed information about the study and affirmed 
Table 1 Characteristics of patients eligible for study and participating in NSABP LTS-01 study
*Total number of eligible patients who are in 65 invited institutions is 2,540

**Total number of participants is 744

***Age is calculated as of November 29, 2006 when the LTS-01 protocol started to accrue patients

\begin{tabular}{|c|c|c|c|}
\hline Characteristics & Eligible* (\%) & Participating** $(\%)$ & $\begin{array}{l}\text { (\%) Participating/ } \\
\text { Eligible }\end{array}$ \\
\hline \multicolumn{4}{|l|}{ Gender } \\
\hline Male & $1,408(55.43)$ & 427 (57.39) & 30.33 \\
\hline Female & $1,132(44.57)$ & $317(42.61)$ & 28.00 \\
\hline \multicolumn{4}{|l|}{ Protocol } \\
\hline C-05 & $651(25.63)$ & $157(21.10)$ & 24.12 \\
\hline C-06 & $630(24.80)$ & $186(25.00)$ & 29.52 \\
\hline $\mathrm{C}-07$ & $1,038(40.87)$ & $372(50.00)$ & 35.84 \\
\hline $\mathrm{R}-02$ & $153(6.02)$ & $15(2.02)$ & 9.80 \\
\hline $\mathrm{R}-03$ & $68(2.68)$ & $14(1.88)$ & 20.59 \\
\hline \multicolumn{4}{|l|}{ Disease stage } \\
\hline 0 Nodes & $1,118(44.02)$ & $312(41.94)$ & 27.91 \\
\hline 1-3 Nodes & $1,044(41.10)$ & $319(42.88)$ & 30.56 \\
\hline $4+$ Nodes & $369(14.53)$ & $111(14.92)$ & 30.08 \\
\hline Unknown & $9(0.35)$ & $2(0.27)$ & 22.22 \\
\hline \multicolumn{4}{|l|}{$\operatorname{Age}^{* * *}$} \\
\hline$\leq 59$ & $696(27.40)$ & $212(28.49)$ & 30.46 \\
\hline $60-69$ & $713(28.07)$ & $242(32.53)$ & 33.94 \\
\hline$\geq 70$ & $1131(44.53)$ & $290(38.98)$ & 25.64 \\
\hline Mean \pm SD & $66.60 \pm 11.55$ & $65.61 \pm 10.53$ & \\
\hline \multicolumn{4}{|l|}{ Race } \\
\hline White or Hispanic & $2,283(89.88)$ & $697(93.68)$ & 30.53 \\
\hline Black & $138(5.43)$ & $26(3.49)$ & 18.84 \\
\hline Other & $115(4.53)$ & $20(2.69)$ & 17.39 \\
\hline Unknown & $4(0.16)$ & $1(0.13)$ & 25.00 \\
\hline \multicolumn{4}{|l|}{ Institution type } \\
\hline Main & $1,062(41.81)$ & $301(40.46)$ & 28.34 \\
\hline Satellite & $1,088(42.83)$ & $361(48.52)$ & 33.18 \\
\hline Missing & $390(15.35)$ & $82(11.02)$ & 21.03 \\
\hline
\end{tabular}

the subject's consent to participate. If the subject affirmed their consent, the interviewer proceeded with the interview $(n=708)$. If a subject withdrew their consent, they were marked as a refusal $(n=12)$. In three cases, subjects were deemed ineligible at phone contact due to the following reasons: inability to conduct the interview independently, inability to conduct the interview in English, and having a hearing impairment that hindered the ability to hear the interview over the phone. Subjects who were not reachable within 10 calls or were not reachable due to irreconcilable issues with contact information were considered passive refusals $(n=21)$.

Barriers to institutional recruitment and patient enrollment

Given the large number of potentially eligible patients for this study, we expected to achieve our target enrollment of 1,167 patients during an 18 month period. Over the course of the study, we identified several critical barriers that either delayed or prevented institutional participation. In Table 4 we describe the specific institutional barriers. Over time, we persisted in pursuing institutions that were reluctant to put their applications through the IRB and extended additional financial support to satellite institutions when this was a barrier. One of the unanticipated barriers was that many of the patients at each site had been accrued from satellite institutions, which required additional IRB approvals for each satellite institution to approach patients. The second layer of barriers related to the ability to identify and contact the potentially eligible survivors. There was also considerable institutional variability in the yield of patients for whom contact was obtained, in relationship to the number of potential participants (See Fig. 4).

\section{Discussion}

There is increasing interest in the late effects of specific treatments for cancer among adult cancer survivors. Some studies have used large administrative databases, such as 
Table 2 Characteristics of patients with registration screening form available*

\begin{tabular}{|c|c|c|c|c|c|}
\hline \multicolumn{2}{|l|}{ Characteristics } & Total** (\%) & Agree to participate (\%) & $\begin{array}{l}\text { Not agree to } \\
\text { participate }(\%)\end{array}$ & $\begin{array}{l}\text { Fisher's exact } \\
\text { test } P \text { value }\end{array}$ \\
\hline \multicolumn{6}{|l|}{$\operatorname{Age}^{* * *}$} \\
\hline \multicolumn{2}{|l|}{$\leq 59$} & $236(25.82)$ & $212(28.49)$ & $24(14.12)$ & $<0.0001$ \\
\hline \multicolumn{2}{|l|}{$60-69$} & $289(31.62)$ & $242(32.53)$ & $47(27.65)$ & \\
\hline \multicolumn{2}{|l|}{$\geq 70$} & $389(42.56)$ & $290(38.98)$ & $99(58.24)$ & \\
\hline \multicolumn{2}{|l|}{ Mean \pm SD } & $66.48 \pm 10.56$ & $65.61 \pm 10.53$ & $70.28 \pm 9.83$ & \\
\hline \multicolumn{6}{|l|}{ Marital status } \\
\hline \multicolumn{2}{|l|}{ Married } & $651(72.58)$ & $543(73.98)$ & $108(66.26)$ & 0.052 \\
\hline \multicolumn{2}{|l|}{ Single, Widowed } & $246(27.42)$ & $191(26.02)$ & $55(33.74)$ & \\
\hline \multicolumn{2}{|l|}{ Missing } & 17 & 10 & 7 & \\
\hline \multicolumn{6}{|l|}{ Gender } \\
\hline \multicolumn{2}{|l|}{ Male } & $513(58.76)$ & $417(58.82)$ & $96(58.54)$ & 1.000 \\
\hline \multicolumn{2}{|l|}{ Female } & $360(41.24)$ & $292(41.18)$ & $68(41.46)$ & \\
\hline \multicolumn{2}{|l|}{ Missing } & 41 & 35 & 6 & \\
\hline \multicolumn{6}{|c|}{ First year of diagnosis } \\
\hline \multicolumn{2}{|l|}{$1985-89$} & $13(1.45)$ & $8(1.09)$ & $5(3.07)$ & 0.018 \\
\hline \multicolumn{2}{|l|}{ 1990-94 } & $197(22.04)$ & $164(22.44)$ & $33(20.25)$ & \\
\hline \multicolumn{2}{|l|}{$1995-99$} & $251(28.08)$ & $193(26.40)$ & $58(35.58)$ & \\
\hline \multicolumn{2}{|l|}{$2000-04$} & $433(48.43)$ & $366(50.07)$ & $67(41.10)$ & \\
\hline \multicolumn{2}{|l|}{ Missing } & 20 & 13 & 7 & \\
\hline \multicolumn{6}{|l|}{ Ethnicity } \\
\hline \multicolumn{2}{|l|}{ Hispanic or Latino } & $22(2.74)$ & $18(2.73)$ & $4(2.82)$ & 1.000 \\
\hline \multicolumn{2}{|l|}{ Not } & $780(97.26)$ & $642(97.27)$ & $138(97.18)$ & \\
\hline \multicolumn{2}{|l|}{ Unknown } & 112 & 84 & 28 & \\
\hline \multicolumn{6}{|l|}{ Race\# } \\
\hline \multirow[t]{2}{*}{ White } & Yes & $848(94.01)$ & $692(94.15)$ & $156(93.41)$ & 0.718 \\
\hline & No & $54(5.99)$ & $43(5.85)$ & $11(6.59)$ & \\
\hline \multirow[t]{2}{*}{ Black } & Yes & $30(3.33)$ & $25(3.40)$ & $5(2.99)$ & 1.000 \\
\hline & No & 872 (96.67) & $710(96.60)$ & $162(97.01)$ & \\
\hline \multirow[t]{2}{*}{ Other } & Yes & $30(3.33)$ & $24(3.27)$ & $6(3.59)$ & 0.812 \\
\hline & No & $872(96.67)$ & $711(96.73)$ & $161(96.41)$ & \\
\hline \multirow[t]{2}{*}{ Unknown } & Yes & $12(1.31)$ & $9(1.21)$ & $3(1.76)$ & 0.475 \\
\hline & No & 902 (98.69) & 735 (98.79) & $167(98.24)$ & \\
\hline
\end{tabular}

*Patients having record of date of birth are included in this table

**Total number of patients is 914

***Age is calculated as of November 29, 2006 when the LTS-01 protocol started to accrue patients

\#Some patients might have more than one race

Table 3 Reasons for non-participation on registration screening form

\begin{tabular}{lc}
\hline Reasons* & Number**(\%) \\
\hline Too busy & $22(9.48)$ \\
Experience with cancer too personal/painful & $18(7.76)$ \\
Just not interested & $72(31.03)$ \\
Other & $134(57.76)$ \\
\hline
\end{tabular}

* Some patients have more than one reason

**Total number of non-participants is 232 the SEER-Medicare database, that merge tumor registry identified cases with medical claims data to infer outcomes from specific therapies, such as the risk for later cardiac diagnoses after earlier treatment exposure to anthracyclines $[13,14]$. The strength of such studies is the large sample size and potential for generalizability to the population at large; however, a major limitation is that these results are observational, do not account for selection bias in who gets specific cancer treatments, and inference is made from coded diagnoses from billing documents rather than from 
Table 4 Institutional barriers to study participation

\section{IRB issues}

- Issues with content of consent form and/or other study documents

- Central site and satellites - the need for multiple IRBs

Site resources/staffing

- Study not a priority relative to other studies

- Coordinator transitions delay study progress

- Satellites not interested in participating - also impedes central site's interest to participate

- Change in principal investigator at a site, no one with an interest in participating

Trial administration issues

- Satellites had trouble identifying eligible patients on NSABP website or obtaining eligible patient information from the central site

- Sites are multiple and spread out, so follow-up is perceived to be difficult

- Time since site's last contact with patients has been very long, so follow up is perceived to be difficult

clinical information in the medical record. These studies have no data on drug doses or regimen, which may be critical to understanding late effects. In contrast, studies that have attempted to link tumor registry samples to abstracted medical record data often have greater accuracy in describing late effects, especially when there may be a dose-response relationship to late effects $[15,16]$.

Recruitment of long-term survivors directly from phase III clinical trials databases has great appeal, since specific treatments are assigned at random as part of the study design, and thus the interaction of the treatment exposure with patient characteristics is less likely to be biased. An important limitation is the highly selective nature of clinical trial participants in terms of trial eligibility (e.g., normal kidney, liver function, limited number of comorbid conditions), as well as the selected nature of the institutions and patients willing to participate. Nevertheless, this may be the most effective way to examine the late effects of specific treatment exposures. Unfortunately, most clinical trial protocols only monitor toxicity during treatment and do not record late outcomes other than cancer recurrence and/or death.

Increasingly, various clinical trials groups have made an effort to obtain long-term data on survivors who were previously treated on a clinical trial. For example, Champion et al. [17] recently reported on the quality of life outcomes in survivors of dysgerminoma recruited from several Gynecological Oncology Group (GOG) and MD Anderson Cancer Center trials. There were 238 potentially eligible women identified from the GOG database, but only 171 (72\%) were from currently active GOG clinical sites. Of these, only 142 were available to contact and 117 participated in the survey study, representing about $50 \%$ of those eligible. A similar recruitment result occurred for the small number of patients treated at MD Anderson Cancer Center. The participants were a median of 10.2 years from diagnosis.

In a study of long-term survivors of breast cancer survivors treated on CALGB 8541, Paskett et al. [18]
Fig. 4 Rate of successful accrual by number of eligible patients for 60 institutions with IRB approval.

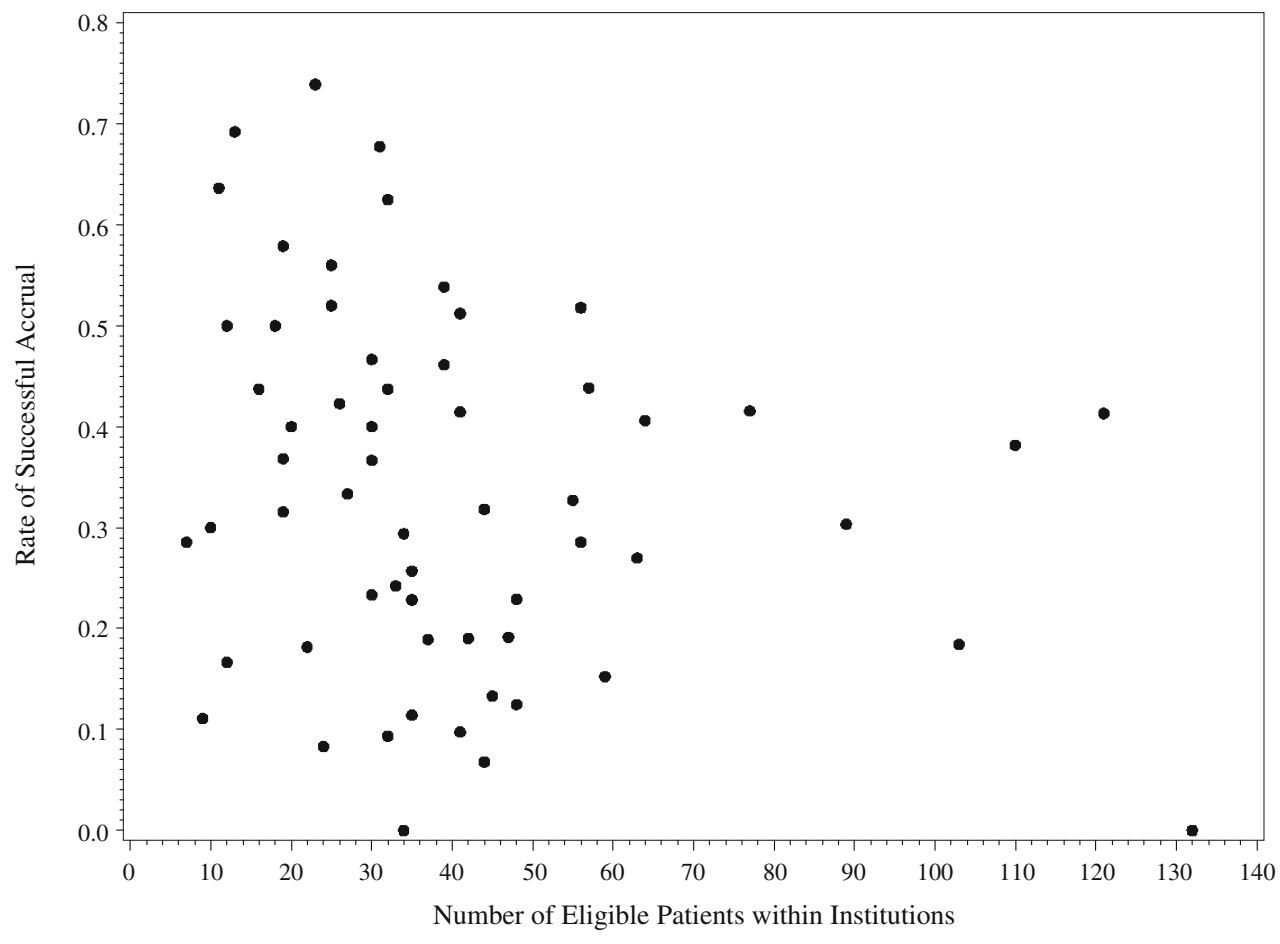

* Each Dot Presents an Institution 
identified 618 survivors who were potentially eligible, having been treated 9.4-16.5 years earlier. Among these, $245(40 \%)$ completed the survey study. The reasons for non-accrual included $10 \%$ of patients were from nonCALGB institutions, $10 \%$ were lost due to institutions not opening the survivorship study, in $13 \%$ of cases the institution did not return the eligibility form, $26 \%$ of the sample who were screened were not eligible, 5\% were not invited by the physician, 16\% refused, and 7\% did not complete the survey [18]. Thus, there were many challenges obtaining participation from these survivors so many years after initial treatment. While the data gathered in this way are useful, they may be biased towards participation of the healthiest long-term survivors. For example, as noted in our study, non-participants tended to be older, not married and treated during an earlier time period. Such comparison data were not available in the study by Paskett et al. [18].

In another recent study from the Southwest Oncology Group (SWOG), Ganz et al. [19] reported on the late cardiac effects of breast cancer patients treated on SWOG 8897, which compared two different adjuvant chemotherapy treatments for breast cancer-one containing doxorubicin. This study required participation in a cardiac evaluation with MUGA scan, as well as medical history evaluation focused on cardiac events. Patients had to be reconsented for this follow-up study, which occurred 510 years after the initial clinical trial randomization. In their study, 1,178 women survivors were available for recruitment to the study and only 180 were registered. Many SWOG institutions did not open the trial and thus for $69 \%$ of the potential study sample, no attempt was made to contact them to invite participation in the cardiac follow-up study. In 13\%, administrative and institutional barriers were responsible for failure to contact potentially eligible subjects. Ineligibility, patient refusal and other reasons accounted for the other non-participants. While this study provided valuable comparison data on the two treatment regimens, the low participation rate limits generalizability to the entire trial sample [19].

Learning from these experiences with recruitment challenges in this setting, we designed the LTS-01 study to provide as much administrative and financial support as possible to encourage the NSABP institutions to open the study and recruit the potentially eligible patients. In addition, we selected the study sites with the largest numbers of eligible survivors, with the idea that it would not be attractive for sites with fewer than 10 patients to obtain IRB approval. We also lessened the burden on the sites by collecting the patient reported outcome data by telephone at a centralized location. In spite of these efforts designed to anticipate the challenges to institutional and patient recruitment, we fell short of our goal of recruiting about $50 \%$ of eligible of patients.
Some of the new findings from this report are that patients assigned to a single institution had in fact been treated at satellites as well as the main institution, presenting an administrative challenge in terms of number of IRB approvals required. A related observation was that some institutions had new clinical and research leadership that had no personal relationship with patients treated 10 15 years earlier. Finally, the older age of the patient population who are colon and rectal cancer survivors was an important patient-related barrier to recruitment. About $45 \%$ of the potentially eligible patients were $\geq 70$ years of age at the time they were approached for participation in LTS-01. Comorbid illness, frailty, hearing problems and lack of interest were frequently cited as reasons for nonparticipation. Patients treated more recently (e.g., on C-07 clinical trial) were more likely to be participants in LTS-01, probably reflecting a personal relationship with the clinical site staff where they were treated, and also being younger. Also, some patients expressed lack of interest in participating due to the lower treatment outcome impact of the earlier trials.

What should be done in the future to facilitate longterm follow-up studies of patients treated on cancer clinical trials? With the growing number of cancer survivors and the limited database on the specific late effects of cancer treatment, there will be an increasing need to provide future patients with information about late effects and long term outcomes of treatment. This is especially warranted if two treatment strategies have similar survival outcomes but differing late effects. Furthermore, if a late effect is common and mutable, high risk individuals could benefit from late effects screening. To this end, we suggest that the NCI should consider providing support and a mechanism for prospective longterm outcome follow-up in selected clinical trials in adults, to follow participants prospectively at regular intervals after treatment, and to maintain some regular contact. This needs to be built into the initial consent process and clinical sites will need to be provided with adequate financial support to maintain contact with participants. Identification of next of kin or other key contacts, as is routinely done in long-term epidemiologic studies, will allow better estimation of biases in loss to follow-up and non-participation. Furthermore, allowing the cooperative group to function as a registry for future survivorship studies would shorten the steps necessary to re-contact patients and invite them to participate in these late effects studies. Engaging patients and survivors in the importance of this aspect of the treatment study will also be important. Investment in this aspect of the clinical trial research agenda should be a high priority, as it is very costly, in terms of personnel and funding to try to locate survivors many years after a hiatus in contact. 
Acknowledgements We thank the staff at the NSABP Operations Center (Barbara Harkins, RN and Teresa Bradley, PhD), the NSABP clinical site coordinators and physicians, and the patients who participated in this study. This research was funded by the American Cancer Society grant RSGPB-05-236-01-CPPB, and through Public Health Service Grants U10-CA-12027, U10-CA-69651, U10-CA37377, and U10-CA-69974 from the National Cancer Institute, Department of Health and Human Services.

Open Access This article is distributed under the terms of the Creative Commons Attribution Noncommercial License which permits any noncommercial use, distribution, and reproduction in any medium, provided the original author(s) and source are credited.

\section{Reference}

1. Hewitt M, Greenfield S, Stovall E. From Cancer Patient to Cancer Survivor: Lost in Transition. Washington, D.C: The National Academies; 2006.

2. Bower JE, Ganz PA, Desmond KA, Rowland JH, Meyerowitz BE, Belin TR. Fatigue in breast cancer survivors: occurrence, correlates, and impact on quality of life. J Clin Oncol. 2000;18(4):743-53.

3. Dow KH, Ferrell BR, Leigh S, Ly J, Gulasekaram P. An evaluation of the quality of life among long-term survivors of breast cancer. Breast Cancer Res Treat. 1996;39(3):261-73. doi:10.1007/BF01806154.

4. Ganz PA, Greendale GA, Petersen L, Kahn B, Bower JE. Breast Cancer in Younger Women: Reproductive and Late Health Effects of Treatment. J Clin Oncol. 2003;21(22):4184-93. doi:10.1200/ JCO.2003.04.196.

5. Ramsey SD, Berry K, Moinpour C, Giedzinska A, Andersen MR. Quality of life in long term survivors of colorectal cancer. Am J gastroenterol. 2002;97(5):1228-34. doi:10.1111/j.1572-0241. 2002.05694.x.

6. Janov AJ, Anderson J, Cella DF, Zuckerman E, Kornblith AB, Holland JC, et al. Pregnancy outcome in survivors of advanced Hodgkin disease. Cancer. 1992;70(3):688-92. doi:10.1002/10970142(19920801)70:3<688::AID-CNCR2820700325>3.0.CO;2-V.

7. Kornblith AB, Anderson J, Cella DF, Tross S, Zuckerman E, Cherin E, et al. Comparison of psychosocial adaptation and sexual function of survivors of advanced Hodgkin disease treated by MOPP, ABVD, or MOPP alternating with ABVD. Cancer. 1992;70(10):2508-16. doi:10.1002/1097-0142(19921115) 70:10<2508::AID-CNCR2820701020>3.0.CO;2-V.

8. Tross S, Herndon J, Korzun A, Kornblith AB, Cella DF, Holland $\mathrm{JF}$, et al. Psychological symptoms and disease-free and overall survival in women with stage II breast cancer. Cancer and Leukemia Group B. J Natl Cancer Inst. 1996;88(10)):661-67. doi:10.1093/jnci/88.10.661.

9. Ganz PA, Hussey MA, Moinpour CM, Unger JM, Hutchins LF, Dakhil SR et al. Late Cardiac Effects of Adjuvant Chemotherapy in Breast Cancer Survivors Treated on Southwest Oncology Group Protocol S8897. J Clin Oncol 2008;JCO.

10. Ramsey SD, Andersen MR, Etzioni R, Moinpour C, Peacock S, Potosky A, et al. Quality of life in survivors of colorectal carcinoma. Cancer. 2000;88(6):1294-1303. doi:10.1002/(SICI)1097-0142 (20000315)88:6<1294::AID-CNCR4>3.0.CO;2-M.

11. Schag CA, Ganz PA, Wing DS, Sim MS, Lee JJ. Quality of life in adult survivors of lung, colon and prostate cancer. Qual Life Res. 1994;3(2):127-41. doi:10.1007/BF00435256.

12. Andersen RM. Revisiting the behavioral model and access to medical care: does it matter? J Health Soc Behav. 1995;36(1):110. doi: $10.2307 / 2137284$

13. Giordano SH, Duan Z, Kuo YF, Hortobagyi GN, Goodwin JS. Use and Outcomes of Adjuvant Chemotherapy in Older Women With Breast Cancer. J Clin Oncol. 2006;24(18):2750-56. doi:10.1200/JCO.2005.02.3028.

14. Patt DA, Goodwin JS, Kuo YF, Freeman JL, Zhang DD, Buchholz TA, et al. Cardiac Morbidity of Adjuvant Radiotherapy for Breast Cancer. J Clin Oncol. 2005;23(30):7475-82. doi:10.1200/JCO.2005.13.755.

15. van Leeuwen FE, Klokman WJ, Veer MB, Hagenbeek A, Krol ADG, Vetter UAO, et al. Long-Term Risk of Second Malignancy in Survivors of HodgkinÆs Disease Treated During Adolescence or Young Adulthood. J Clin Oncol. 2000;18(3):487.

16. van den Belt-Dusebout A, de Wit R, Gietema JA, Horenblas S, Louwman MWJ, Ribot JG, et al. Treatment-Specific Risks of Second Malignancies and Cardiovascular Disease in 5-Year Survivors of Testicular Cancer. J Clin Oncol. 2007;25(28):437078. doi:10.1200/JCO.2006.10.5296.

17. Champion V, Williams SD, Miller A, Reuille KM, Wagler-Ziner K, Monahan PO, et al. Quality of life in long-term survivors of ovarian germ cell tumors: A Gynecologic Oncology Group Study. Gynecol Oncol. 2007;105(3):687-94. doi:10.1016/j.ygyno.2007.01.042.

18. Paskett ED, Herndon JE, Day JM, Stark NN, Winer EP, Grubbs SS, et al. Applying a conceptual model for examining healthrelated quality of life in long-term breast cancer survivors: CALGB study 79804. Psycho-Oncol. 2008;17(11):1108-20. doi:10.1002/pon.1329.

19. Ganz PA, Hussey MA, Moinpour CM, Unger JM, Hutchins LF, Dakhil SR, et al. Late Cardiac Effects of Adjuvant Chemotherapy in Breast Cancer Survivors Treated on Southwest Oncology Group Protocol S8897. J Clin Oncol. 2008;26(8):1223-30. doi:10.1200/JCO.2007.11.8877. 